\title{
Adverse Event Management
}

\author{
Aline Gonzalez Vigani \\ Department of Viral Hepatitis Studies, State University of Campinas (Unicamp); Campinas, SP, Brazil
}

The treatment of hepatitis $\mathrm{C}$ involves combining conventional interferon (IFN) or pegylated IFN (PEG-IFN) with ribavirin (RBV). These therapeutic regimens are associated with numerous adverse events, among which constitutional and neuropsychiatric symptoms, as well as hematological abnormalities, stand out [1,2]. The adverse events observed with the use of PEG-IFN or conventional IFN are similar, and the frequencies of those events are shown in Table 1 [3-5]. Adverse events resulting from the treatment of hepatitis $C$ can jeopardize the quality of life of patients and their response to treatment. The control of those events involves medicinal and non-medicinal interventions. The latter include a reduction in the dosage of IFN or RBV and discontinuation of the treatment.

A temporary or permanent reduction in the dosage of PEGIFN, conventional IFN or RBV as a result of an adverse event is necessary in approximately $30 \%$ of patients. In $10 \%$ of patients it is necessary to discontinue the treatment [4]. Hematological abnormalities (neutropenia, anemia and thrombocytopenia) and depression are the most common causes of dose reduction.

The maintenance of the dosages of medications used in the treatment of hepatitis $\mathrm{C}$ and of the recommended course of treatment are important for the effectiveness of the therapy,

Table 1. Adverse events associated with using the pegylated interferon-ribavirin combination in the treatment of hepatitis $\mathrm{C}$

\begin{tabular}{lc}
\hline Adverse event & $\begin{array}{c}\text { Approximate } \\
\text { incidence (\%) }\end{array}$ \\
\hline Reaction at the site of injection & $36-58$ \\
Fatigue & $50-64$ \\
Headache & $50-62$ \\
Myalgia & $42-56$ \\
Fever & $43-56$ \\
Chills & $24-48$ \\
Alopeica & $28-36$ \\
Artralgia & $27-35$ \\
Irritability & $24-35$ \\
Depression & $21-34$ \\
Anorexia & $14-32$ \\
Dermatitis & $16-21$ \\
Anemia & $12-22$ \\
Neutropenia & $17-20$ \\
Thrombocytopenia & $3-6$ \\
\hline
\end{tabular}

The Brazilian Journal of Infectious Diseases $\quad$ 2007;11 (5) Suppl. 1:66-70. (C) 2007 by The Brazilian Journal of Infectious Diseases and Contexto Publishing. All rights reserved. as shown in Figure 1 [6]. Dose reduction is associated with lowering the rate at which a sustained viral response (SVR) is achieved. Early identification and strategies for controlling adverse events are important in the prevention of moderate and severe complications. These practices also attenuate the deleterious effects on the quality of life of patients and maximize the effectiveness of treatment for hepatitis C.

\section{Constitutional Symptoms}

Constitutional symptoms such as fatigue, headache and myalgia are the most common adverse events in patients

Figure 1. Effect of dose reduction of pegylated interferon (PEG-IFN) and ribavirin (RBV) in relation to the sustained viral response (SVR) rate.

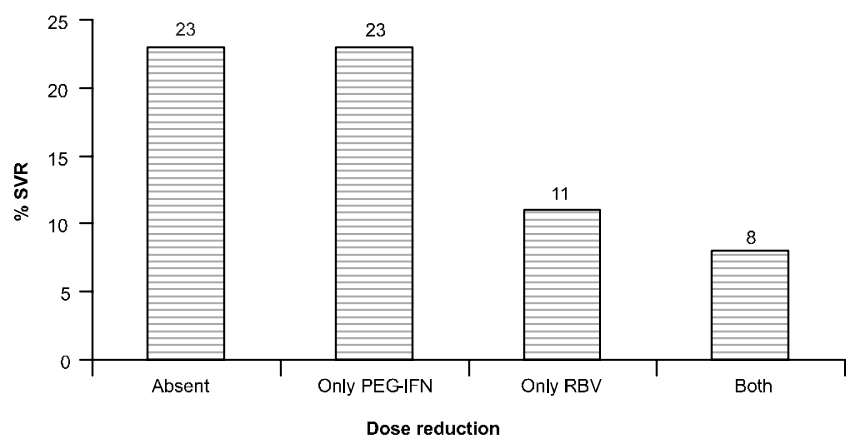

treated with the combination of IFN and RBV (Table 1). Practically all patients manifest at least one such event during the administration of the initial doses of IFN. These symptoms typically disappear or become less intense after the first month of treatment. Paracetamol, acetaminophen and ibuprofen taken immediately before the injection of IFN alleviate the symptoms. Adequate hydration, together with light to moderate exercise, can help minimize these side effects.

\section{Hematological Effects}

In approximately $25 \%$ of patients, it is necessary at least to reduce the dosage of IFN or RBV due to abnormalities in laboratory test results [4]. Conventional IFN and PEG-IFN have both been associated with the suppression of hematopoiesis. In addition, hemolytic anemia occurs in all patients treated with RBV, although the intensity of that anemia is variable.

\section{Anemia}

Anemia resulting from the treatment of hepatitis $C$ is a multifactorial side effect. Those factors increase the destruction of red blood cells and reduce their production. The increased destruction of red blood cells occurs due to hemolysis triggered by RBV, and the reduction in red blood 
cell production occurs due to IFN-induced suppression of bone marrow erythroid precursors [7,8].

The RBV penetrates erythrocytes, in which monophosphate, diphosphate and triphosphate are phosphorylated. Those pharmacologically active forms are incapable of passing through the erythrocyte membrane, and so remain intracellularly retained at a concentration 60 times greater than that of plasma $[7,8]$. The accumulated phosphorylated derivates are slowly eliminated from the erythrocytes, which have a half-life of 40 days. In contrast, the elimination of RBV from plasma is rapid, since it has a halflife of 24 hours.

The mechanisms by which IFN can exacerbate RBVinduced anemia include suppression of hematopoiesis in bone marrow and an increase in the destruction of erythroid precursor cells [9].

Anemia starts and develops almost immediately after the initiation of therapy, becoming more intense after 4 to 6 weeks of treatment. The average decrease in the level of hemoglobin $(\mathrm{Hb})$ is 2.5 to $3.0 \mathrm{~g} / \mathrm{dL}$, but more intense degrees of hemolysis can occur. Anemia associated with combined therapy can exacerbate other side effects resulting from the treatment of hepatitis $\mathrm{C}$ such as dyspnea, fatigue, dizziness and headache.

Non-pharmacological control of the treatment of anemia involves dose reduction or permanent discontinuation of RBV [9]. In patients without heart disease, a reduction in the dosage of ribavirin when the levels of $\mathrm{Hb}$ fall below $10 \mathrm{~g} / \mathrm{dL}$ is recommended, and discontinuation is recommended when $\mathrm{Hb}$ levels are under $8.5 \mathrm{~g} / \mathrm{dL}$ (Table 2) [10]. Those recommendations are more restricted to patients with stable heart disease. In rare cases, when the level of $\mathrm{Hb}$ is lower than $8.5 \mathrm{~g} / \mathrm{dL}$, a transfusion of packed red blood cells, in conjunction with discontinuation of the treatment for hepatitis C, might be necessary.

Between 9 and 22\% of patients receiving combined treatment for hepatitis $\mathrm{C}$ require an $\mathrm{RBV}$ dose reduction due to anemia $[3,4]$.

However, the strategy of RBV dose reduction has been associated with a modest increase in the level of $\mathrm{Hb}(\sim 1 \mathrm{~g} / \mathrm{dL})$ at 4-8 weeks after the reduction, as well as with a decrease in the rate at which an SVR is achieved (Figure 1) [6,11].

Hematopoietic growth factors such as alpha erythropoietin can be an alternative in relation to RBV dose reduction for the treatment of anemia arising during the treatment of hepatitis C. Alpha erythropoietin is a synthetic glycoprotein that has a mechanism of action similar to that of endogenous erythropoietin, which is a hormone produced by the renal peritubular capillaries that stimulates erythropoiesis in bone marrow.

Studies indicate that therapy with erythropoietin at the dosage of 40,000 IU once a week elevates the level of Hb after one week of use and thus permits the maintenance of RBV dosage in patients that become anemic during combined therapy for hepatitis $C[12,13]$. In the study conducted by Afdhal et al., the initial dose of RBV was maintained in $88 \%$ of the patients who received erythropoietin during treatment for hepatitis C, and Hb levels increased (from 10.6 \pm 0.9 to $13.2 \pm$ $1.2 \mathrm{~g} / \mathrm{dL}$ ) in those same patients [13]. The initial dose of RBV was maintained in $66 \%$ of the patients who received a placebo. The cost of alpha erythropoietin is high, but it is a therapeutic option for patients who develop an $\mathrm{Hb}$ level $<10 \mathrm{~g} / \mathrm{dL}$ during treatment of hepatitis C [14].

\section{Neutropenia}

A decrease in neutrophil counts to below normal values occurs in the majority of patients receiving conventional IFN or PEG-IFN and results from the suppression of bone marrow caused by those medications [15]. Neutropenia (neutrophil count $<750$ cells/mL) is more common with the use of PEGIFN than with that of conventional IFN, with values of $20 \%$ and $4 \%$, respectively $[3,4]$. Intense neutropenia ( $<500$ cells/ $\mathrm{mm}^{3}$ ) is also more common in patients treated with PEG-IFN than in those treated with conventional IFN, with values of $5 \%$ and $1 \%$, respectively.

After a single injection of PEG-IFN, neutrophil counts decrease, on average, 21\% within the first 24 hours but generally stabilize at higher levels thereafter. Therefore, the measurement of neutrophil counts should be carried out before the administration of IFN so that more reliable values can be obtained.

In patients who develop neutropenia during treatment for hepatitis C, in general, IFN dose reduction or discontinuation of treatment is necessary. Recommendations for dose reduction or discontinuation of the treatment due to neutropenia are shown in Table 2. Neutrophil counts generally return to pretreatment levels 2 to 4 weeks after the end of treatment [2].

The clinical implications of IFN-related neutropenia are not associated with an increased risk of infectious complications $[16,17]$. The low infection rates observed in neutropenic patients undergoing treatment for hepatitis $C$ can reflect an early reduction in IFN dosage and, in addition, can indicate that those patients have a lower risk of infection when compared with neutropenic patients undergoing chemotherapy.

Filgrastim is beginning to be used as an adjuvant therapy, at the dose of $300 \mu \mathrm{g} 1$ to 3 times a week, to increase neutrophil counts in patients who have less than 750 cells $/ \mathrm{mm}^{3}$ during treatment for hepatitis C. However, clinical experience with filgrastim in such cases is still limited $[10,15]$. Filgrastim is a granulocyte-colony stimulating factor structurally similar to that produced by human cells and obtained through genetic engineering. Filgrastim interacts with receptors on the surface of myeloid progenitor cells in bone marrow, inducing proliferation, differentiation and activation of functional cells.

\section{Thrombocytopenia}

Thrombocytopenia observed in patients undergoing treatment for hepatitis $\mathrm{C}$ is a consequence of IFN-induced 
Table 2. Dose modifications for adverse events resulting from therapy with interferon and ribavirin according to manufacturer recommendations

\begin{tabular}{|c|c|c|c|c|c|}
\hline \multicolumn{2}{|c|}{ Hematological parameters } & \multirow[t]{2}{*}{ PEG-IFN- $\alpha 2 b$} & \multirow[t]{2}{*}{ PEG-IFN- $\alpha 2 a$} & \multirow[t]{2}{*}{ IFN } & \multirow{2}{*}{$\begin{array}{l}\text { RBV } \\
\text { Reduce to } 11 \mathrm{mg} / \mathrm{kg} \\
\text { Discontinue }\end{array}$} \\
\hline Hemoglobin ${ }^{\mathrm{a}}$ & $\begin{array}{l}<10 \mathrm{~g} / \mathrm{dL} \\
<8.5 \mathrm{~g} / \mathrm{dL}\end{array}$ & & & & \\
\hline $\begin{array}{l}\text { Platelets } \\
\text { Neutrophils }\end{array}$ & $\begin{array}{l}<25,000 / \mathrm{mm}^{3} \\
<750 / \mathrm{mm}^{3}\end{array}$ & $\begin{array}{l}\text { Discontinue } \\
\text { Reduce to } \\
1 \mu \mathrm{g} / \mathrm{kg}\end{array}$ & $\begin{array}{l}\text { Discontinue } \\
\text { Reduce to } \\
135 \mu \mathrm{g}\end{array}$ & $\begin{array}{l}\text { Discontinue } \\
\text { Reduce by } 50 \%\end{array}$ & \\
\hline & $<500 / \mathrm{mm}^{3}$ & Discontinue & Discontinue & Discontinue & Discontinue \\
\hline
\end{tabular}

Figure. 2. Algorithm for the pharmacological treatment of INF-induced depression.

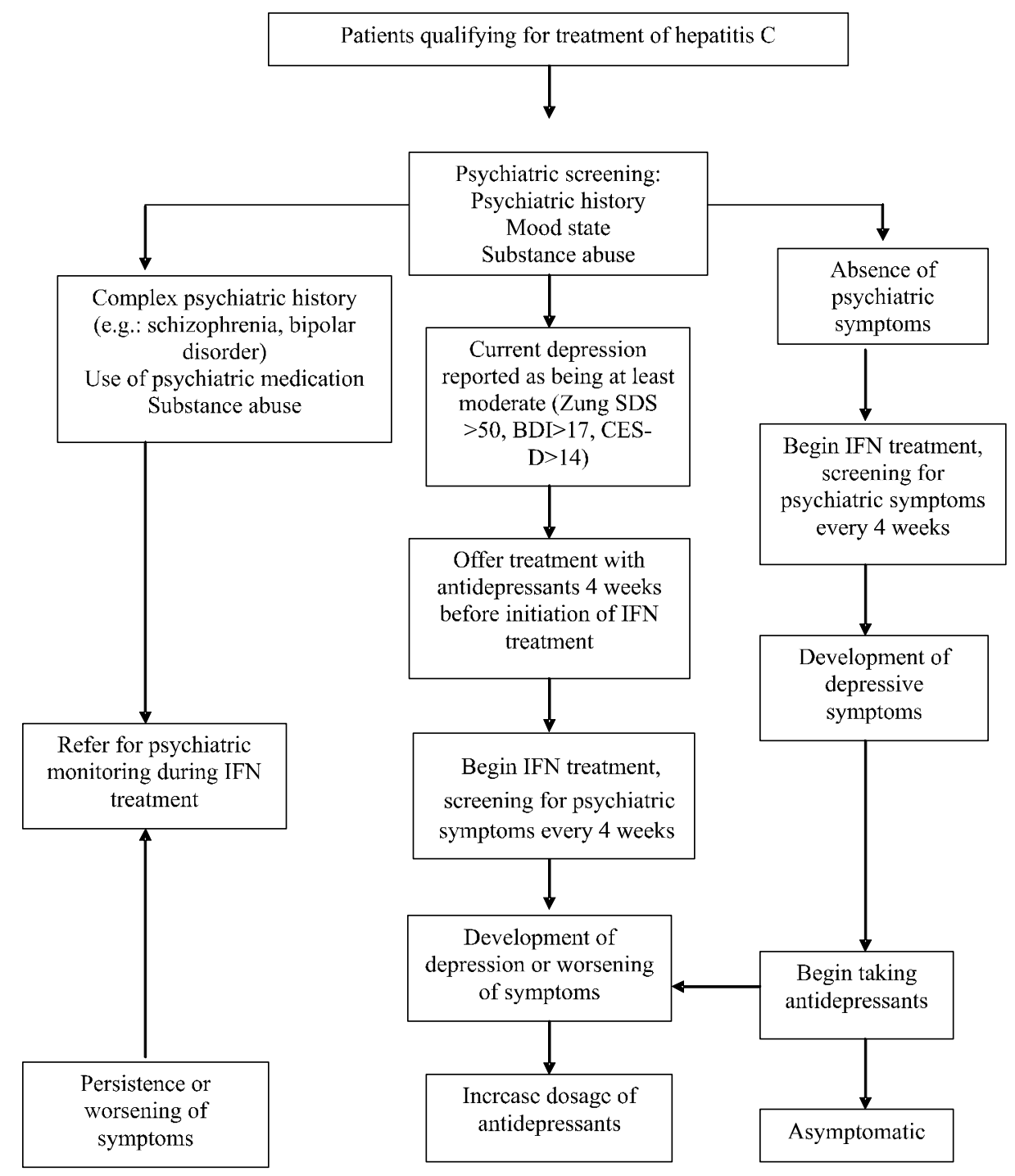

BDI=Beck Depression Inventory; CES-D=Center for Epidemiological Studies Depression Rating Scale; Zung SDS=Zung Self Rating Depression Scale. 
bone marrow suppression. A drop in platelet counts during combined treatment for hepatitis $\mathrm{C}$ is common, but IFN dose reduction or discontinuation of treatment as a result of that drop is uncommon [2,3]. Thrombocytopenia requiring IFN dose reduction is more common in patients treated with PEGIFN and RBV than in those treated with conventional IFN and $\mathrm{RBV}$, with frequency rates of $4 \%$ and $1 \%$, respectively [18].

Recommendations for the discontinuation of PEG-IFN or conventional IFN as a result of thrombocytopenia are shown in Table 2. Platelet counts generally return to pretreatment levels by 4 weeks after the discontinuation of treatment [2]. There are ongoing studies that aim to evaluate the effectiveness and safety of growth factors that stimulate the production and maturation of megakaryocytes in thrombocytopenic patients undergoing treatment for hepatitis $\mathrm{C}$.

Rare cases of autoimmune thrombocytopenic purpura have been described during the treatment for hepatitis $\mathrm{C}$, and the presence of that disorder should be investigated in rare cases involving an intense decrease in platelet counts.

\section{Neuropsychiatric Symptoms}

Approximately 20\% to $30 \%$ of patients report depressive symptoms during treatment for hepatitis C [19]. The control of depression in patients who are candidates to receive treatment for hepatitis $C$ is shown in Figure 2 [20]. In the pretreatment phase, it is important to establish the psychiatric status of the patient, which involves determining whether there is a history of depression, assessing the mood state and identifying abuse of illegal substances. Patients who abuse drugs or who have a complex psychiatric history involving disorders such as schizophrenia and bipolar disorder or who are receiving psychiatric treatment should be jointly accompanied by a psychiatrist during treatment for hepatitis C. Patients with current or previous depression should be evaluated before the initiation of treatment for hepatitis $\mathrm{C}$, preferably through the use of scales that infer the intensity of depression. In patients with moderate depression, antidepressant medication can be given 4 weeks before the initiation of treatment for hepatitis $C$.

Antidepressants belonging to a class of selective serotonin reuptake inhibitors (SSRIs) constitute the treatment of choice for IFN-associated depression [19]. Such antidepressants are safe and well-tolerated in patients with hepatic disease. In addition, the treatment success rate in patients with depression during treatment for hepatitis $\mathrm{C}$ is near 90\% [18]. Fluoxetine, sertraline, citalopram, paroxetine and other SSRIs can have a slightly sedative effect, and the choice of SSRI to be used should be based on that effect and on the predominant symptomatology of the patient [21]. In patients with fatigue or cognitive slowness, fluoxetine or sertraline can be preferred over paroxetine, which tends to be less stimulating. In case of suicidal ideation or attempted suicide, the treatment of hepatitis $\mathrm{C}$ should be immediately suspended [22]. The dose of antidepressant medication started during treatment of hepatitis $\mathrm{C}$ should be slowly reduced over a 6 to 12 month period after the end of the treatment.

\section{Thyroid Disease}

Approximately 1-6\% of individuals treated with IFN develop thyroid abnormalities [23]. In all patients, an evaluation of thyroid function is recommended. Levels of thyroid-stimulating hormone should be determined before the initiation of treatment for hepatitis C, every 12 weeks during treatment and once after the end of the treatment. Individuals who develop hypothyroidism while undergoing treatment should receive hormonal replacement therapy.

\section{Pulmonary Side Effects}

Dry cough, which can occur during treatment, has been associated with the use of RBV. In most cases the cough is tolerable, but occasionally it is necessary to discontinue the use of RBV. Cases in which cough becomes productive or is accompanied by abnormal pulmonary auscultation findings or fever, pneumonia should be investigated. Interstitial pneumonia can be severe, but it is normally reversible with the discontinuation of therapy [19].

\section{Retinopathy}

The use of IFN can trigger or aggravate prior retinopathy. Subconjunctival hemorrhage and retinal hemorrhage have been reported during treatment with IFN [19]. Patients with risk factors for retinopathy such as systemic arterial hypertension and diabetes mellitus should undergo ophthalmological examination before and during therapy under the supervision of an ophthalmologist. Treatment should be discontinued in individuals who present either retinal lesions during treatment or the worsening of a prior lesion.

\section{References}

1. Maddrey W.C. Safety of combination interferon alfa-2b/ribavirin therapy in chronic hepatitis C-relapsed and treatment-naive patients. Semin Liver Dis 1999;19 Suppl 1:67-75.

2. Fried M.W. Side effects of therapy of hepatitis $C$ and their management. Hepatology 2002;36(5 Suppl 1):S237-44.

3. Manns M.P., McHutchison J.G., Gordon S.C., et al. Peginterferon alfa-2b plus ribavirin compared with interferon alfa-2b plus ribavirin for initial treatment of chronic hepatitis $C$ : a randomised trial. Lancet 2001;22:958-65.

4. Fried M.W., Hadziyannis S.J. Treatment of chronic hepatitis C infection with peginterferons plus ribavirin. Semin Liver Dis 2004;24 Suppl 2:47-54.

5. Hadziyannis S.J., Sette H. Jr., Morgan T.R., et al. Peginterferonalpha2a and ribavirin combination therapy in chronic hepatitis C: a randomized study of treatment duration and ribavirin dose. Ann Intern Med 2004;2;140(5):346-55.

6. Shiffman M.L., Di Bisceglie A.M., Lindsay K.L., et al. Hepatitis C Antiviral Long-Term Treatment Against Cirrhosis Trial Group. Peginterferon alfa-2a and ribavirin in patients with chronic hepatitis $\mathrm{C}$ who have failed prior treatment. Gastroenterology 2004;126(4):1015-23.

7. Bodenheimer H.C. Jr., Lindsay K.L., Davis G.L., et al. Tolerance and efficacy of oral ribavirin treatment of chronic hepatitis C: a multicenter trial. Hepatology 1997;26(2):473-7.

8. De Franceschi L., Fattovich G., Turrini F., et al. Hemolytic anemia induced by ribavirin therapy in patients with chronic hepatitis $C$ virus infection: role of membrane oxidative damage. Hepatology 2000;31(4):997-1004. 
9. Kowdley K.V. Hematologic side effects of interferon and ribavirin therapy. J Clin Gastroenterol 2005;39(1 Suppl):S3-8.

10. Afdhal N.H. Role of epoetin alfa in maintaining ribavirin dose. Gastroenterol Clin North Am 2004;33(1 Suppl):S25-35.

11. Sulkowski M.S., Wasserman R., Brooks L., et al. Changes in haemoglobin during interferon alpha-2b plus ribavirin combination therapy for chronic hepatitis $\mathrm{C}$ virus infection. $\mathrm{J}$ Viral Hepat 2004;11(3):243-50.

12. Dieterich D.T., Wasserman R., Brau N., et al. Once-weekly epoetin alfa improves anemia and facilitates maintenance of ribavirin dosing in hepatitis $C$ virus-infected patients receiving ribavirin plus interferon alfa. Am J Gastroenterol 2003;98(11):2491-9.

13. Afdhal N.H., Dieterich D.T., Pockros P.J., et al. Epoetin alfa maintains ribavirin dose in HCV-infected patients: a prospective, double-blind, randomized controlled study. Gastroenterology 2004;126(5):1302-11.

14. Devine E.B., Kowdley K.V., Veenstra D.L., Sullivan S.D. Management strategies for ribavirin-induced hemolytic anemia in the treatment of hepatitis $\mathrm{C}$ : clinical and economic implications. Value Health 2001;4(5):376-84.

15. Collantes R.S., Younossi Z.M. The use of growth factors to manage the hematologic side effects of PEG-interferon alfa and ribavirin. J Clin Gastroenterol 2005;39(1 Suppl):S9-13.
16. Sulkowski M.S. Management of the hematologic complications of hepatitis C therapy. Clin Liver Dis 2005;9(4):601-16.

17. Soza A., Everhart J.E., Ghany M.G., et al. Neutropenia during combination therapy of interferon alfa and ribavirin for chronic hepatitis C. Hepatology 2002;36(5):1273-9.

18. Aspinall R.J., Pockros P.J. The management of side-effects during therapy for hepatitis C. Aliment Pharmacol Ther 2004;20(9):917-29.

19. Russo M.W., Fried M.W. Side effects of therapy for chronic hepatitis C. Gastroenterology 2003;124(6):1711-9.

20. Raison C.L., Demetrashvili M., Capuron L., Miller A.H. Neuropsychiatric adverse effects of interferon-alpha: recognition and management. CNS Drugs 2005;19(2):105-23.

21. Edwards J.G., Anderson I. Systematic review and guide to selection of selective serotonin reuptake inhibitors. Drugs 1999;57(4):507-33.

22. Janssen H.L., Brouwer J.T., van der Mast R.C., Schalm S.W. Suicide associated with alfa-interferon therapy for chronic viral hepatitis. J Hepatol 1994;21(2):241-3.

23. Huang M.J., Tsai S.L., Huang B.Y., et al. Prevalence and significance of thyroid autoantibodies in patients with chronic hepatitis $\mathrm{C}$ virus infection: a prospective controlled study. Clin Endocrinol (Oxf) 1999;50(4):503-9. 